\title{
Antimagic orientation of graphs with minimum degree at least 33
}

\author{
Songling Shan \\ Illinos State University, Normal,IL 61790 \\ sshan12@ilstu.edu
}

May 4, 2020

\begin{abstract}
An antimagic labeling of a directed graph $D$ with $n$ vertices and $m$ arcs is a bijection from the set of arcs of $D$ to the integers $\{1, \cdots, m\}$ such that all $n$ oriented vertex sums are pairwise distinct, where an oriented vertex sum is the sum of labels of all arcs entering that vertex minus the sum of labels of all arcs leaving it. A graph $G$ has an antimagic orientation if it has an orientation which admits an antimagic labeling. Hefetz, Mütze, and Schwartz conjectured that every connected graph admits an antimagic orientation. In this paper, we show that every bipartite graph without both isolated and degree 2 vertices admits an antimagic orientation and every graph $G$ with $\delta(G) \geq 33$ admits an antimagic orientation. Our proof relies on a newly developed structural property of bipartite graphs, which might be of independent interest.
\end{abstract}

Keywords: Labeling; Antimagic labeling; Antimagic orientation; Matching

\section{Introduction}

All graphs considered are simple and finite unless otherwise stated. For two integers $p, q$, $[p, q]:=\{p, p+1 \ldots, q\}$ if $q \geq p$, and $[p, q]:=\emptyset$ if $q<p$. A labeling of a graph $G$ with $m$ edges is a bijection from $E(G)$ to a set $S$ of $m$ integers, and the vertex sum at a vertex $v \in V(G)$ is the sum of labels on the edges incident to $v$. A labeling is antimagic if $S=[1, m]$ and all the vertex sums are distinct. A graph is antimagic if it has an antimagic labeling.

Hartsfield and Ringel [7] introduced antimagic labelings in 1990 and conjectured that every connected graph other than $K_{2}$ is antimagic. There have been some significant progress towards this conjecture. Let $G$ be a graph with $n$ vertices other than $K_{2}$. In 2004, Alon, Kaplan, Lev, Roditty, and Yuster [1] showed that there exists a constant $c$ such that if $G$ has minimum degree at least $c \cdot \log n$, then $G$ is antimagic. They also proved that $G$ is antimagic when the maximum degree of $G$ is at least $n-2$, and they proved that all complete multipartite graphs (other than $K_{2}$ ) are antimagic. The latter result of Alon et al. was improved by Yilma [18] in 2013.

Apart from the results above on dense graphs, the antimagic labeling conjecture has been also verified for regular graphs. Started with Cranston [4] showing that every bipartite regular graph is antimagic, regular graphs of odd degree [5], and finally all regular graphs [2, 
3] were shown to be antimatic sequentially. For more results on the antimagic labeling conjecture for other classes of graphs, see $[6,8,10,11]$.

Hefetz, Mütze, and Schwartz [9] introduced the variation of antimagic labelings, i.e., antimagic labelings on directed graphs. An antimagic labeling of a directed graph with $m$ arcs is a bijection from the set of arcs to the integers $\{1, \ldots, m\}$ such that any two oriented vertex sums are pairwise distinct, where an oriented vertex sum is the sum of labels of all arcs entering that vertex minus the sum of labels of all arcs leaving it. A digraph is called antimagic if it admits an antimagic labeling. For an undirected graph $G$, if it has an orientation such that the orientation is antimagic, then we say $G$ admits an antimagic orientation. Hefetz, Mütze, and Schwartz in the same paper posted the following problems.

Question 1 ([9]). Is every connected directed graph with at least 4 vertices antimagic?

Conjecture 2 ([9]). Every connected graph admits an antimagic orientation.

Hefetz, Mütze, and Schwartz [9] showed that every orientation of a dense graph is antimagic and almost all regular graphs have an antimagic orientation. Particulary, they showed that every orientation of stars (other than $K_{1,2}$ ), wheels, and complete graphs (other than $K_{3}$ ) is antimagic. Conjecture 2 has been also verified for regular graphs $[9,12,14,16]$, biregular bipartite graphs with minimum degree at least two [13], Halin graphs [19], graphs with large maximum degree [17], and graphs with large independence number [15]. In this paper, by supporting Conjecture 2, we obtain the results below.

Theorem 3. Every bipartite graph with no vertex of degree 0 or 2 admits an antimagic orientation.

Theorem 4. Every graph $G$ with $\delta(G) \geq 33$ admits an antimagic orientation.

The remainder of this paper is organized as follows. We introduce several preliminary results in Section 2. In Section 3, we prove Theorem 3, and in Section 4, we prove Theorem 4.

\section{Notation and Preliminary Lemmas}

Let $G$ be a graph. We use $e(G)$ for $|E(G)|$. For $S \subseteq V(G), G[S]$ is the subgraph of $G$ induced by $S$. For two disjoint subsets $S, T \subseteq V(G)$, we denote by $E_{G}(S, T)$ the set of edges in $G$ with one endvertex in $S$ and the other in $T$, and let $e_{G}(S, T)=\left|E_{G}(S, T)\right|$. If $G$ is bipartite with partite sets $X$ and $Y$, we denote $G$ by $G[X, Y]$ to emphasis the bipartitions. Given an orientation $D$ of $G$, a labeling $\sigma$ on $A(D)$ that is the set of $\operatorname{arcs}$ of $D$, and a vertex $v \in V(D)$, we use $s_{[D, \sigma]}(v)$ to denote the oriented sum at $v$ in $D$, which is the sum of labels of all arcs entering $v$ minus the sum of labels of all arcs leaving it in the digraph $D$.

For a matching $M$ of $G$, we use $V(M)$ to denote the set of vertices saturated by $M$. For a vertex $x \in V(M), M(x)$ is the vertex that is matched to $x$ in $M$. For each subset $X \subseteq V(M)$, if $X$ is an independent set in $M$, then $M(X)$ is the set of of vertices that are matched to vertices from $X$ in $M$. By this definition, $|X|=|M(X)|$ and $X$ and $M(X)$ are disjoint. An $M$-augmenting path is a path whose edges are alternating between edges in $M$ and edges not in $M$ and with both endpoints being not saturated by $M$.

A trail is an alternating sequence of vertices and edges $v_{0} e_{1} v_{1} \ldots e_{t} v_{t}$ such that $v_{i-1}$ and $v_{i}$ are the endvertices of $e_{i}$, for each $i \in[1, t]$, and the edges are all distinct (but there might 
be repetitions among the vertices). A trail is closed if $v_{0}=v_{t}$, and is open otherwise. An Euler tour of $G$ is a closed trail in $G$ that contains all the edges of $G$. We will need the following classic result of Euler in proving a lemma later on.

Theorem 5 (Euler, 1736). A mutigraph $G$ has an Euler tour if and only if $G$ has at most one nontrivial component and every vertex of $G$ has an even degree.

Lemma 6 ( [11]). Let $t, n$ be integers with $t \geq 1$ and $n \geq 2$, and let $n=r_{1}+\ldots+r_{t}$ be a partition of $n$, where $r_{i}$ is an integer that is at least 2 for each $i \in[1, t]$. Then the set $\{1, \ldots, n\}$ can be partitioned into pairwise disjoint subsets $R_{1}, \ldots, R_{t}$ such that for each $i \in[1, t],\left|R_{i}\right|=r_{i}$ and $\sum_{r \in R_{i}} r \equiv 0(\bmod n+1)$ if $n$ is even, and $\sum_{r \in R_{i}} r \equiv 0(\bmod n)$ if $n$ is odd.

The following result was proved in [15] without the furthermore part. However, the furthermore part is easy to obtain by following the same proof of Lemma 2.2 in [15] by just letting vertices in $T$ to be not the endvertices of the edge-disjoint trails that decompose $E(G)$, which can be definitely guaranteed by the conditions imposed on $T$. So we omit the proof.

Lemma 7. Let $p, m$ be integers with with $p \geq 0, m \geq 1$, and let $G$ be a graph with $m$ edges. Then there exist an orientation $D$ of $G$ and a bijections $\sigma: A(D) \rightarrow\{p+1, \ldots, p+m\}$ such that for each $v \in V(G)$,

$$
-(p+m)+\left\lfloor\frac{d_{G}(v)-1}{2}\right\rfloor \leq s_{[D, \sigma]}(v) \leq\left\lfloor\frac{d_{G}(v)-1}{2}\right\rfloor+(p+m) .
$$

Furthermore, for $T \subseteq V(G)$ and each $v \in T$, if $d_{G}(v)$ is even and $N_{G}(v) \cap(V(G) \backslash T) \neq \emptyset$, then we can choose $\sigma$ so that $s_{[D, \sigma]}(v)=\frac{d_{G}(v)}{2}$.

Lemma 8. Let $p, m$ be integers with $p \geq 0$ and $m \geq 1$, and let $G[S, T]$ be a bipartite graph with $m$ edges such that every vertex from $T$ has an even degree in $G$ (so $m$ is even). If $m \equiv 0(\bmod 4)$, let $\delta_{m}=p+m$; and if $m \equiv 2(\bmod 4)$, let $\delta_{m}=p+m+1$. Then there exist an orientation $D$ of $G$ and a bijection $\sigma: A(D) \rightarrow\{p+1, \ldots, p+m-1\} \cup\left\{\delta_{m}\right\}$ such that

$$
\begin{aligned}
& s_{[D, \sigma]}(v)=-d_{G}(v) \quad \text { for each } v \in T \text {, and } \\
& \left\lfloor\frac{d_{G}(v)-1}{2}\right\rfloor-\delta_{m} \leq s_{[D, \sigma]}(v) \leq\left\lfloor\frac{d_{G}(v)-1}{2}\right\rfloor+\delta_{m} \quad \text { for each } v \in S .
\end{aligned}
$$

Proof. Suppose $G$ has in total $2 \ell$ vertices of odd degree for some integer $\ell \geq 0$. We obtain a new graph $G^{*}$ by pairing up these vertices into $\ell$ pairs, and for each pair, adding an edge joining the two vertices. Note that $G^{*}=G$ if $\ell=0$.

Each component of $G^{*}$ has an Euler tour by Theorem 5. By deleting all the edges in $E\left(G^{*}\right) \backslash E(G)$, we partition all edges of $G$ into $\ell$ trails $T_{1}, T_{2}, \ldots, T_{\ell}$ (each $T_{i}$ is either open or closed). For each $i \in[1, \ell]$, let

$$
T_{i}=x_{t_{i-1}+1} e_{t_{i-1}+1} y_{t_{i-1}+1} f_{t_{i-1}+1} x_{t_{i-1}+2} \ldots y_{t_{i}-1} f_{t_{i}-1} x_{t_{i}}
$$


where $t_{0}=0$. Note that $t_{m}-1=m / 2$ and $\left|E\left(T_{i}\right)\right|=2\left(t_{i}-1-t_{i-1}\right)$. Since for every $v \in T$, $d_{G}(v)$ is even, we can further assume that in each $T_{i}$, for each $j \in\left[t_{i-1}+1, t_{i}\right]$,

$$
x_{j} \in S \quad \text { and } \quad y_{j} \in T \text {. }
$$

Also by the construction of $T_{i}$ 's, each vertex from $S$ is the endvertex of at most one open trail.

For each $j \in[1, m / 2-1]$, we direct each edge $e_{j}$ from $x_{j}$ to $y_{j}$, and direct each edge $f_{j}$ from $y_{j}$ to $x_{j+1}$. Denote by $D$ this orientation of $G$.

If $m \equiv 0(\bmod 4)$, for each $i \in[1, m / 4]$, let

$$
\begin{aligned}
\sigma\left(e_{2 i-1}\right) & =4 i-3, & \sigma\left(f_{2 i-1}\right) & =4 i-1 ; \\
\sigma\left(e_{2 i}\right) & =4 i-2, & \sigma\left(f_{2 i}\right) & =4 i .
\end{aligned}
$$

If $m \equiv 2(\bmod 4)$, let

$$
\begin{aligned}
\sigma\left(e_{2 i-1}\right) & =4 i-3, \quad \sigma\left(f_{2 i-1}\right)=4 i-1 \quad \text { for each } i \in\left[1, \frac{m+2}{4}\right] ; \\
\sigma\left(e_{2 i}\right) & =4 i-2, \quad \sigma\left(f_{2 i}\right) \quad=4 i \quad \text { for each } i \in\left[1, \frac{m-2}{4}\right] .
\end{aligned}
$$

By the definition of $\sigma$ above, for each $j \in[1, m / 2], e_{j}, f_{j}$ contributes -2 to the vertex sum at $y_{j}$ that is shared by $e_{j}$ and $f_{j}$. Since for each vertex $y \in T$, the edges incident to $y$ in $G$ are partitioned into $\frac{d_{G}(y)}{2}$ pairs of edges in the form of $e_{j}, f_{j}$, it holds $s_{[D, \sigma]}(y)=-d_{G}(y)$.

For each $j \in[1, m / 2-1], f_{j}, e_{j+1}$ contributes 1 to the vertex sum at $x_{j+1}$ that is shared by $f_{j}$ and $e_{j+1}$. For each vertex $x \in S$, the edges incident to $x$ in $G$ are partitioned into at least $\left\lfloor\frac{d_{G}(x)-1}{2}\right\rfloor$ pairs of edges in the form of $f_{j}, e_{j+1}$. If $d_{G}(x)$ is odd, then $x$ is the endvertex of exactly one open trails in $\left\{T_{1}, T_{2}, \ldots, T_{\ell}\right\}$. Thus, the edge incident to $x$ not counted in the pairs $f_{j}, e_{j+1}$ has a label in $\left[-\delta_{m}, \delta_{m}\right]$. If $d_{G}(x)$ is even, then $x$ can be the endvertices of at most one closed trails in $\left\{T_{1}, T_{2}, \ldots, T_{\ell}\right\}$. Thus, the two edges incident to $x$ not counted in the pairs $f_{j}, e_{j+1}$ have a label in $\left[-\delta_{m}, \delta_{m}\right]$ : one is negative and the other is positive, which add up to a value in $\left[-\delta_{m}, \delta_{m}\right]$. Hence, for each $x \in S$, it holds $\left\lfloor\frac{d_{G}(x)-1}{2}\right\rfloor-\delta_{m} \leq s_{[D, \sigma]}(x) \leq\left\lfloor\frac{d_{G}(x)-1}{2}\right\rfloor+\delta_{m}$. This finishes the proof of Lemma 8.

The following result on bipartite graphs is heavily used in our proofs, which might be of independent interest to other applications also.

Lemma 9. If $G$ is a bipartite graph, then $V(G)$ has a partition $S \cup T$ that satisfies the following conditions:

(a) $G$ has a matching $M$ with $M \subseteq E_{G}(S, T)$ and $M$ saturates $S$;

(b) $T$ is an independent set in $G$.

Proof. It suffices to prove the statement only for every component of $G$. Thus we may assume that $G$ is connected. Let $[X, Y]$ be a bipartition of $G$. Assume, without loss of 
generality, that $|X| \leq|Y|$. Let $M$ be a matching of $G$ that saturates the largest number of vertices from $X$. We will find a desired partition $S \cup T$ of $V(G)$ based on $X$ and $Y$.

If $X \backslash V(M)=\emptyset$, then we are done by letting $S=X$ and $T=Y$. Thus, $X \backslash V(M) \neq \emptyset$. Let

$$
\begin{aligned}
X_{1} & =X \cap V(M), & X_{0} & =X \backslash X_{1}, \\
Y_{1} & =Y \cap V(M), & Y_{0} & =Y \backslash Y_{1} .
\end{aligned}
$$

Since $|X \cap V(M)|=|Y \cap V(M)|$ and $|Y| \geq|X|, X_{0} \neq \emptyset$ implies $Y_{0} \neq \emptyset$. By the maximality of $M$, it holds

$$
E_{G}\left(X_{0}, Y_{0}\right)=\emptyset
$$

Let

$$
B_{0}=N_{G}\left(X_{0}\right), \quad A_{0}=M\left(B_{0}\right), \quad C_{0}=N_{G}\left(Y_{0}\right), \quad D_{0}=M\left(C_{0}\right) .
$$

Clearly, $B_{0}, C_{0} \neq \emptyset$ as $G$ is connected. For each integer $i$ with $i \geq 1$, define

$$
B_{i}=N_{G}\left(A_{i-1}\right) \backslash \bigcup_{j=0}^{i-1} B_{j}, \quad A_{i}=M\left(B_{i}\right) .
$$

Let

$$
B=\cup_{i=0}^{\infty} B_{i}, \quad A=\cup_{i=0}^{\infty} A_{i} .
$$

By the definition, $B_{i} \cap B_{j}=\emptyset$ and $A_{i} \cap A_{j}=\emptyset$ for every pair of $i, j$ with $i, j \geq 0$ and $i \neq j$. Since $\left|A_{i}\right|=\left|B_{i}\right|$ for each $i$ with $i \geq 0$, it holds

$$
|A|=|B|
$$

Let

$$
X_{r}=X_{1} \backslash A, \quad Y_{r}=Y_{1} \backslash B
$$

By the definition of $A$,

$$
E_{G}\left(A, Y_{r}\right)=\emptyset
$$

Let

$$
S=B \cup X_{r}, \quad T=A \cup Y_{r} \cup X_{0} \cup Y_{0} .
$$

It is left to show that $S \cup T$ is a desired partition of $V(G)$. Since $|A|=|B|$ by (2), $|S|=\left|X_{1}\right|$. Furthermore, by the definitions of $A$ and $B, A=M(B)$, and consequently $X_{r}=M\left(Y_{r}\right)$. Thus, $M$ is still a matching in $G$ that saturates $S$ and has size $|S|$, and $M \subseteq E_{G}(S, T)$. We only show that $T$ is an independent in $G$. As each of $A, Y_{r}, X_{0}$ and $Y_{0}$ is an independent set in $G,(1)$ and (3), respectively, implies that $A \cup Y_{r}$ and $X_{0} \cup Y_{0}$ are independent sets in $G$. Since $E_{G}\left(X_{0}, A\right)=\emptyset$ and $E_{G}\left(X_{0}, Y_{r}\right)=\emptyset$ by $N_{G}\left(X_{0}\right)=B_{0} \subseteq B$, $A \cup Y_{r} \cup X_{0}$ is an independent set in $G$. Since $E_{G}\left(Y_{0}, Y_{r}\right)=\emptyset$ and $E_{G}\left(Y_{0}, X_{0}\right)=\emptyset$ by (1), we are only left to show that $E_{G}\left(Y_{0}, A\right)=\emptyset$.

It suffices to only show that $D_{0} \subseteq Y_{r}$. Since $D_{0} \subseteq Y_{r}$ implies that $C_{0} \subseteq X_{r}$ by the definitions of the sets $A$ and $B$, and $C_{0} \subseteq X_{r}$ implies that $C_{0} \cap A=\emptyset$, which yields $E_{G}\left(Y_{0}, A\right)=\emptyset$. 
To show $D_{0} \subseteq Y_{r}$, we just show that for each $i$ with $i \geq 0, E_{G}\left(A_{i}, D_{0}\right)=\emptyset$. Assume to the contrary and let $k$ be the smallest index such that $E_{G}\left(A_{k}, D_{0}\right) \neq \emptyset$. Let $d_{0} \in D_{0}$ and $a_{k} \in A_{k}$ such that $d_{0} a_{k} \in E(G), b_{k}=M\left(a_{k}\right), a_{k-1} \in A_{k-1}$ such that $a_{k-1} b_{k} \in E(G)$. In general, for each $i=k-1, k-2, \ldots, 1$, let

$$
b_{i}=M\left(a_{i}\right), \quad a_{i-1} \in A_{i-1} \quad \text { such that } a_{i-1} b_{i} \in E(G) .
$$

Furthermore, let $b_{0}=M\left(a_{0}\right)$ and $x_{0} \in X_{0}$ such that $b_{0} x_{0} \in E(G)$, and $c_{0}=M\left(d_{0}\right)$ and $y_{0} \in Y_{0}$ such that $c_{0} y_{0} \in E(G)$.

Note that for $i, j \in[0, k]$ with $i \neq j, a_{i} \neq a_{j}$ and $b_{i} \neq b_{j}$, as $A_{i} \cap A_{j}=\emptyset$ and $B_{i} \cap B_{j}=\emptyset$. Furthermore, by the minimality of $k, a_{i} \neq d_{0}$ and $b_{i} \neq d_{0}$. Thus

$$
P:=y_{0} c_{0} d_{0} a_{k} b_{k} a_{k-1} b_{k-1} \ldots b_{1} a_{0} b_{0} x_{0}
$$

is an $M$-augmenting path, and $M^{\prime}:=(M \backslash E(P)) \cup(E(P) \backslash M)$ is a matching in $G$ such that $\left|V\left(M^{\prime}\right) \cap X\right|>|V(M) \cap X|$, showing a contradiction to the choice of $M$. Therefore, $E_{G}\left(A_{i}, D_{0}\right)=\emptyset$ for each $i$ with $i \geq 0$. This completes the proof.

\section{Proof of Theorem 3}

Let $S \cup T$ be a partition of $V(G)$ satisfying the requirements in Lemma 9. Let

$$
n_{1}=|S|, \quad n_{2}=|T|, \quad S=\left\{x_{1}, x_{2}, \ldots, x_{n_{1}}\right\}, \quad T=\left\{y_{1}, y_{2}, \ldots, y_{n_{2}}\right\} .
$$

Assume, without loss of generality, that

$$
M=\left\{x_{1} y_{1}, x_{2} y_{2}, \ldots, x_{n_{1}} y_{n_{1}}\right\} .
$$

. For each $i \in\left[n_{1}+1, n_{2}\right]$, let $e_{i}$ be an edge incident to $y_{i}$ in $G$, and let

$$
M^{*}=M \cup\left\{e_{n_{1}+1}, \ldots, e_{n_{2}}\right\} .
$$

In other words, each vertex from $T$ is incident to one and exactly one edge from $M^{*}$. Furthermore, let

$$
H=G-E(G[S])-M^{*}, \quad m_{1}=|E(G[S])|, \quad m_{2}=e(H) .
$$

Clearly, $m_{1}+m_{2}+\left|M^{*}\right|=m_{1}+m_{2}+n_{2}=m:=e(G)$. Let $T_{1}=\left\{y \in Y: d_{G}(y)=1\right\}$ and $t_{1}=\left|T_{1}\right|$. Assume, without loss of generality, that $T_{1}=\left\{y_{n_{2}-t_{1}+1}, y_{n_{2}-t_{1}+2}, \ldots, y_{n_{2}}\right\}$. Clearly, $d_{H}\left(y_{i}\right)=0$ for each $i \in\left[n_{2}-t+1, n_{2}\right]$. We consider two cases below regarding how large $n_{2}$ is.

Case 1: $n_{2} \leq m_{2}$.

This case basically follows the same idea as in the Proof of Theorem 1.5 in [15], but we repeat the process for self-completeness.

We give an orientation $D$ of $G$ and a labeling $\sigma$ of $A(D)$ through four parts below. 
(1) Orient and label $H$ : direct each edge from $T$ to $S$. For each $i \in\left[1, n_{2}-t_{1}\right]$, let $A_{i}$ be the set of all edges incident to $y_{i}$ in $H$. Clearly, $\left|A_{1}\right|+\left|A_{2}\right|+\ldots+\left|A_{n_{2}-t_{1}}\right|=m_{2}$. Since $G$ has no vertex of degree 2 or isolated vertex, $\left|A_{i}\right| \geq 2$. By applying Lemma 6 to $m_{2}$ with $t=n_{2}-t_{1}$ and $r_{i}=\left|A_{i}\right|$ for each $i \in[1, t]$, the set $\left\{1,2, \ldots, m_{2}\right\}$ can be partitioned into $R_{1}, R_{2}, \ldots, R_{n_{2}-t_{1}}$ such that for each $i \in\left[1, n_{2}-t_{1}\right],\left|R_{i}\right|=\left|A_{i}\right|$ and $\sum_{r \in R_{i}} r \equiv 0$ $\left(\bmod m_{2}\right)$ if $m_{2}$ is even, and $\sum_{r \in R_{i}} r \equiv 0\left(\bmod m_{2}\right)$ if $m_{2}$ is odd. Label edges in $A_{i}$ by integers in $R_{i}$ in an arbitrary way as long as distinct edges receive distinct labels.

(2) Orient and label $G[S]$ : applying Lemma 7 to get the orientation and labeling with $p=m_{2}$ and $m=m_{1}$;

(3) Orient and label $M^{*} \backslash M=\left\{e_{n_{1}+1}, \ldots, e_{n_{2}}\right\}$ : direct each edge from $T$ to $S$, and for each $i \in\left[n_{1}+1, n_{2}\right]$, assign $m_{1}+m_{2}+\left(i-n_{1}\right)$ to $e_{i}$.

Let $D^{*}$ be the union of the digraphs obtained through the three parts above, and $\sigma^{*}$ be the labeling on $A\left(D^{*}\right)$ consists of the three labelings above. Assume that the sums at vertices from $S=\left\{x_{1}, \ldots, x_{n_{1}}\right\}$ satisfy

$$
s_{\left[D^{*}, \sigma^{*}\right]}\left(x_{1}\right) \leq s_{\left[D^{*}, \sigma^{*}\right]}\left(x_{2}\right) \leq \ldots \leq s_{\left[D^{*}, \sigma^{*}\right]}\left(x_{n_{1}}\right) .
$$

(4) Orient and label $M$ : direct each edge from $T$ to $S$, and for each $i \in\left[1, n_{1}\right]$, assign $m_{1}+m_{2}+n_{2}-n_{1}+i$ to $x_{i} y_{i}$.

Let $D$ and $\sigma$ be the resulting orientation and labeling, respectively. It is clear that $\sigma$ is injective. We show that $\sigma$ is an antimagic labeling of $D$. By Step 4, we have

$$
s_{[D, \sigma]}\left(x_{1}\right)<s_{[D, \sigma]}\left(x_{2}\right)<\ldots<s_{[D, \sigma]}\left(x_{n_{1}}\right) .
$$

Furthermore, for each $i \in\left[1, n_{1}\right]$, by Step $2, s_{\left[D^{*}, \sigma^{*}\right]}\left(x_{i}\right) \geq\left\lfloor\frac{d_{G[S]}\left(x_{i}\right)-1}{2}\right\rfloor-m_{1}-m_{2}$, by Steps 3 and 4 , we know $s_{[D, \sigma]}\left(x_{i}\right) \geq s_{\left[D^{*}, \sigma^{*}\right]}\left(x_{i}\right)+m_{1}+m_{2}+n_{2}-n_{1}+i>0$. For each vertex $y_{i} \in T, i \in\left[1, n_{2}\right]$, all the edges incident to $y_{i}$ are oriented towards $S$. Thus, $s_{[D, \sigma]}\left(y_{i}\right)<0$.

Thus, for each $x \in S$ and each $y \in T, s_{[D, \sigma]}(x)>s_{[D, \sigma]}(y)$. Therefore, it is left to only show that all vertices from $T$ have distinct sums under $\sigma$ in $D$.

By Steps 1, 3 and 4, for each $i \in\left[1, n_{2}\right]$ and for some integr $a_{i} \geq 0$, we have

$$
\left|s_{[D, \sigma]}\left(y_{i}\right)\right|= \begin{cases}a_{i} m_{2}+m_{1}+m_{2}+\sigma_{i}, & \text { if } m_{2} \text { is odd } \\ a_{i}\left(m_{2}+1\right)+m_{1}+m_{2}+\sigma_{i}, & \text { if } m_{2} \text { is even }\end{cases}
$$

where $\sigma_{i} \in\left[1, n_{2}\right]$ are all distinct. Since $n_{2} \leq m_{2}$, for any two distinct $i, j \in\left[1, n_{2}\right]$,

$$
s_{[D, \sigma]}\left(y_{i}\right)-s_{[D, \sigma]}\left(y_{j}\right) \not \equiv\left\{\begin{array}{lll}
0 & \left(\bmod m_{2}\right), & \text { if } m_{2} \text { is odd } \\
0 & \left(\bmod m_{2}+1\right), & \text { if } m_{2} \text { is even }
\end{array}\right.
$$

Consequently, $s_{[D, \sigma]}\left(y_{i}\right) \neq s_{[D, \sigma]}\left(y_{j}\right)$. 
The proof for Case 1 is complete.

Case 2: $n_{2} \geq m_{2}+1$.

In this case, we develop a result similar to Lemma 6 but using nonconsecutive integers not necessarily starting at 1 .

For each $i \in\left[1, n_{2}-t_{1}\right]$, let $A_{i}$ be the set of all edges incident to $y_{i}$ in $H$. Clearly, $\left|A_{1}\right|+\left|A_{2}\right|+\ldots+\left|A_{n_{2}-t_{1}}\right|=m_{2}$. Since $G$ has no vertex of degree 2 or isolated vertex, $\left|A_{i}\right| \geq 2$. Let $m_{2}=3 k+2 \ell$, for some integers $k, \ell \geq 0$, where $k$ is the number of sets $A_{i}$ 's with an odd cardinality. We may assume that $k+\ell \geq 1$. Otherwise, we follow the same proof as in Case 1, and the vertex sums at vertices from $T$ will naturally be all distinct since all these vertices have degree 1 in $G$.

Subcase 2.1: $k=0$.

In this case, all $\left|A_{i}\right|$ 's are even. We give an orientation $D$ of $G$ and a labeling $\sigma$ of $A(D)$ through four parts below.

(1) Orient and label $G[S]$ : applying Lemma 7 to get the orientation and labeling with $p=0$ and $m=m_{1}$;

(2) Orient and label $H$ : direct each edge from $T$ to $S$. For each $i$, partition all edges in $A_{i}$ into $\left|A_{i}\right| / 2$ many 2 -element subsets. Thus, we have in total $m_{2} / 2$ many 2-element subsets $B_{1}, B_{2}, \ldots, B_{m_{2} / 2}$ of edges. For each $B_{i}, i \in\left[1, m_{2} / 2\right]$, we assign

$$
m_{1}+n_{2}+i, m-(i-1)
$$

to the two edges from it. By the way above of assigning labels to edges in $A_{i}$ 's, $i \in$ $\left[1, n_{2}-t_{1}\right]$, the sum of labels assigned to edges from each $A_{i}$ is

$$
a_{i}\left(m+m_{1}+n_{2}+1\right) \quad \text { for some integer } a_{i} \geq 1 \text {. }
$$

(3) Orient and label $M^{*} \backslash M=\left\{e_{n_{1}+1}, \ldots, e_{n_{2}}\right\}$ : direct each edge from $T$ to $S$, and for each $i \in\left[n_{1}+1, n_{2}\right]$, assign $m_{1}+\left(i-n_{1}\right)$ to $e_{i}$.

Let $D^{*}$ be the union of the digraphs obtained through the three parts above, and $\sigma^{*}$ be the labeling on $A\left(D^{*}\right)$ consists of the three labelings above. Assume that the sums at vertices from $S=\left\{x_{1}, \ldots, x_{n_{1}}\right\}$ satisfy

$$
s_{\left[D^{*}, \sigma^{*}\right]}\left(x_{1}\right) \leq s_{\left[D^{*}, \sigma^{*}\right]}\left(x_{2}\right) \leq \ldots \leq s_{\left[D^{*}, \sigma^{*}\right]}\left(x_{n_{1}}\right) .
$$

(4) Orient and label $M$ : direct each edge from $T$ to $S$, and for each $i \in\left[1, n_{1}\right]$, assign $m_{1}+n_{2}-n_{1}+i$ to $x_{i} y_{i}$.

Let $D$ and $\sigma$ be the resulting orientation and labeling, respectively. It is clear that $\sigma$ is injective. We show that $\sigma$ is an antimagic labeling of $D$. By Step 4, we have that

$$
s_{[D, \sigma]}\left(x_{1}\right)<s_{[D, \sigma]}\left(x_{2}\right)<\ldots<s_{[D, \sigma]}\left(x_{n_{1}}\right) .
$$


Furthermore, for each $i \in\left[1, n_{1}\right]$, by Lemma 7 and Step $1, s_{\left[D^{*}, \sigma^{*}\right]}\left(x_{i}\right) \geq\left\lfloor\frac{d_{G[S]}\left(x_{i}\right)-1}{2}\right\rfloor-$ $m_{1}$, we know $s_{[D, \sigma]}\left(x_{i}\right) \geq s_{\left[D^{*}, \sigma^{*}\right]}\left(x_{i}\right)+m_{1}+n_{2}-n_{1}+i \geq 0$. For each vertex $y_{i} \in T$, $i \in\left[1, n_{2}\right]$, all the edges incident to $y_{i}$ are oriented towards $S$. Thus, $s_{[D, \sigma]}\left(y_{i}\right)<0$.

Thus, for each $x \in S$ and each $y \in T, s_{[D, \sigma]}(x)>s_{[D, \sigma]}(y)$. Therefore, it is left to only show that all vertices from $T$ have distinct sums under $\sigma$ in $D$.

By Steps 2, 3 and 4, for each $i \in\left[1, n_{2}\right]$, we have

$$
\left|s_{[D, \sigma]}\left(y_{i}\right)\right|=a_{i}\left(m_{1}+n_{1}+m+1\right)+m_{1}+\sigma_{i} \text { for some integer } a_{i} \geq 1,
$$

where $\sigma_{i} \in\left[1, n_{2}\right]$ are all distinct. Since $n_{2}<m_{1}+n_{1}+m+1$, for any two distinct $i, j \in\left[1, n_{2}\right]$,

$$
s_{[D, \sigma]}\left(y_{i}\right)-s_{[D, \sigma]}\left(y_{j}\right) \not \equiv 0 \quad\left(\bmod m_{1}+n_{1}+m+1\right)
$$

Consequently, $s_{[D, \sigma]}\left(y_{i}\right) \neq s_{[D, \sigma]}\left(y_{j}\right)$.

The proof for Subcase 2.1 is complete.

Subcase 2.2: $k \geq 1$.

Recall that $m_{2}=3 k+2 \ell$ and $n_{2} \geq m_{2}+1$. Thus $m \geq n_{2}+m_{2} \geq 6 k+4 \ell+1$, and $m-2 k-\ell+2=m_{2}+n_{2}+m_{1}-2 k-\ell+2>m_{1}+3 k+\ell$. We assume, without loss of generality, that $\left|A_{1}\right|, \ldots,\left|A_{k}\right|$ are odd, and $\left|A_{k+1}\right|, \ldots,\left|A_{n_{2}-t_{1}}\right|$ are all even.

We will use the labels from the set $A=[1, k] \cup\left[m_{1}+k+1, m_{1}+2 k\right] \cup\left[m_{1}+3 k+\right.$ $\left.1, m_{1}+3 k+\ell\right] \cup[m-2 k-\ell+2, m-2 k+1] \cup\{m-2 k+2, m-2 k+4, \ldots, m-2, m\}$ for edges from $A_{i}$ 's. For each $i \in[1, k]$, edges in $A_{i}$ can be partitioned into one 3 -subset, and $\left(\left|A_{i}\right|-3\right) / 2$ many 2 -subsets. For each $i \in\left[k+1, n_{2}-t_{1}\right]$, edges in $A_{i}$ can be partitioned into $\left|A_{i}\right| / 2$ many 2 -subsets. Let $B_{1}, B_{2}, \ldots, B_{k}$ be the $k 3$-sets and $C_{1}, \ldots, C_{\ell}$ be the $\ell$-sets obtained by partition edges from each $A_{i}$ 's. For each $i \in[1, k]$, we assign edges in each $B_{i}$ the following three numbers:

$$
i, \quad m_{1}+k+i, \quad m-2 i+2 .
$$

For each $i \in[1, \ell]$, we assign edges in each $C_{i}$ the following two numbers:

$$
m_{1}+3 k+i, \quad m-2 k+2-i .
$$

By the way above of assigning labels to edges in $A_{i}$ 's, $i \in\left[1, n_{2}-t_{1}\right]$, the sum of labels assigned to edges from each $A_{i}$ is

$$
a_{i}\left(m+m_{1}+k+2\right) \quad \text { for some integer } a_{i} \geq 1 .
$$

We give an orientation $D$ of $G$ and a labeling $\sigma$ of $A(D)$ through four parts below.

(1) Orient and label $G[S]$ : applying Lemma 7 to get the orientation and labeling with $p=k$ and $m=m_{1}$;

(2) Orient and label $H$ : direct each edge from $T$ to $S$. Assign labels in the set $A$ to the edges in $\bigcup_{i=1}^{n_{2}-t_{1}} A_{i}$ as described previously.

Note that the set of unused labels is

$$
\begin{aligned}
& B=\left[m_{1}+2 k+1, m_{1}+3 k\right] \cup\left[m_{1}+3 k+\ell+1, m-2 k-\ell+1\right] \cup\{m-2 k+3, m-2 k+5, \ldots, m-1\}, \\
& \text { and }|B|=k+m-m_{1}-5 k-2 \ell+1+k-1=m_{2}+n_{2}-3 k-2 \ell=n_{2} .
\end{aligned}
$$


(3) Orient and label $M^{*} \backslash M=\left\{e_{n_{1}+1}, \ldots, e_{n_{2}}\right\}$ : direct each edge from $T$ to $S$, and assign the first $n_{2}-n_{1}$ smallest numbers from $B$ to edges in $M^{*} \backslash M$ such that distinct edges receive distinct labels.

Let $D^{*}$ be the union of the digraphs obtained through the three parts above, and $\sigma^{*}$ be the labeling on $A\left(D^{*}\right)$ consists of the three labelings above. Assume that the sums at vertices from $S=\left\{x_{1}, \ldots, x_{n_{1}}\right\}$ satisfy

$$
s_{\left[D^{*}, \sigma^{*}\right]}\left(x_{1}\right) \leq s_{\left[D^{*}, \sigma^{*}\right]}\left(x_{2}\right) \leq \ldots \leq s_{\left[D^{*}, \sigma^{*}\right]}\left(x_{n_{1}}\right) .
$$

(4) Orient and label $M$ : direct each edge from $T$ to $S$, and assign the remaining $n_{2}-\left(n_{2}-\right.$ $\left.n_{1}\right)=n_{1}$ numbers from $B$ to edges in $M$ such that $x_{i} y_{i}$ is assigned with the $i$-th smallest number.

Let $D$ and $\sigma$ be the resulting orientation and labeling, respectively. It is clear that $\sigma$ is injective. We show that $\sigma$ is an antimagic labeling of $D$. By Step 4, we have

$$
s_{[D, \sigma]}\left(x_{1}\right)<s_{[D, \sigma]}\left(x_{2}\right)<\ldots<s_{[D, \sigma]}\left(x_{n_{1}}\right) .
$$

Furthermore, for each $i \in\left[1, n_{1}\right]$, by Lemma 7 and Step $1, s_{\left[D^{*}, \sigma^{*}\right]}\left(x_{i}\right) \geq\left\lfloor\frac{d_{G[S]}\left(x_{i}\right)-1}{2}\right\rfloor-$ $m_{1}-k$, we know $s_{[D, \sigma]}\left(x_{i}\right) \geq s_{\left[D^{*}, \sigma^{*}\right]}\left(x_{i}\right)+m_{1}+2 k+1 \geq 0$. For each vertex $y_{i} \in T$, $i \in\left[1, n_{2}\right]$, all the edges incident to $y_{i}$ are oriented towards $S$. Thus, $s_{[D, \sigma]}\left(y_{i}\right)<0$.

Thus for each $x \in S$ and each $y \in T, s_{[D, \sigma]}(x)>s_{[D, \sigma]}(y)$. Therefore, it is left to only show that all vertices from $T$ have distinct sums under $\sigma$ in $D$.

By Steps 2, 3, 4 and (5), for each $i \in\left[1, n_{2}\right]$, we have

$$
\left|s_{[D, \sigma]}\left(y_{i}\right)\right| \geq a_{i}\left(m+m_{1}+k+2\right)+\sigma_{i} \quad \text { for some integer } a_{i} \geq 0,
$$

where $\sigma_{i} \in B$ are all distinct. Since $\sigma_{i} \leq m-1<m+m_{1}+k+2$, for any two distinct $i, j \in\left[1, n_{2}\right]$,

$$
s_{[D, \sigma]}\left(y_{i}\right)-s_{[D, \sigma]}\left(y_{j}\right) \not \equiv 0 \quad\left(\bmod m+m_{1}+k+2\right)
$$

Consequently, $s_{[D, \sigma]}\left(y_{i}\right) \neq s_{[D, \sigma]}\left(y_{j}\right)$.

The proof for Subcase 2.2 is complete.

\section{Proof of Theorem 4}

Let $L$ be a spanning bipartite subgraph of $G$ with the maximum number of edges. Since $|E(L)|$ is maximum among all spanning bipartite subgraphs of $G$,

$$
d_{L}(v) \geq \frac{d_{G}(v)}{2} \quad \text { for every } v \in V(G) .
$$

By Lemma 9, we let $S \cup T$ be a partition of $V(L)=V(G), M \subseteq E_{L}(S, T)$ be a matching that saturates $S$ and has size $|S|$, and let $L^{*}=L-E(L[S])$ be the spanning bipartite graph of $L$ between $S$ and $T$. 
Let

$$
n_{2}=|S|, \quad n_{1}=|T|, \quad S=\left\{x_{1}, x_{2}, \ldots, x_{n_{2}}\right\}, \quad T=\left\{y_{1}, y_{2}, \ldots, y_{n_{1}}\right\} .
$$

Assume, without loss of generality, that

$$
M=\left\{x_{1} y_{1}, x_{2} y_{2}, \ldots, x_{n_{1}} y_{n_{2}}\right\} .
$$

For each $i \in\left[n_{2}+1, n_{1}\right]$, let $e_{i}$ be an edge incident to $y_{i}$ in $L^{*}$, and let

$$
M^{*}=M \cup\left\{e_{n_{2}+1}, \ldots, e_{n_{1}}\right\} .
$$

In other words, each vertex from $T$ is incident to one and exactly one edge in $M^{*}$. Furthermore, let

$$
H=L^{*}-M^{*}, \quad G_{1}=G-E(H)-M^{*} .
$$

Note that for every vertex $y \in T$,

$$
d_{H}(v)=d_{L}(v)-1 \geq \frac{d_{G}(v)}{2}-1,
$$

and $E\left(G_{1}\right)=E(G) \backslash\left(E(H) \cup M^{*}\right)=E(G[S]) \cup E(G[T]) \cup\left(E_{G}(S, T) \backslash E_{L^{*}}(S, T)\right)$. We now modify $G_{1}$ to get a new graph by adding some edges from $H$ such that in the new graph the degree of every vertex from $T$ is divisible by 4 and that every vertex from $T$ has a neighbor from $S$. Specifically, for each $v \in T$, if $d_{G_{1}}(v) \equiv c(\bmod 4)$, where $c=0,1,2,3$, we take exactly $4-c$ edges incident to $v$ in $H$ and add these $4-c$ edges into $G_{1}$. Call $G_{2}$ the resulting in graph from $G_{1}$, and $H^{\prime}$ the resulting in graph from $H$. From the construction, for each $v \in T$,

$$
d_{G_{2}}(v) \equiv 0 \quad(\bmod 4), \quad d_{H^{\prime}}(v) \geq d_{H}(v)+c-4,
$$

where $c \in\{0,1,2,3\}$ satisfies $d_{G_{1}}(v) \equiv c(\bmod 4)$.

We then split the bipartite graph $H^{\prime}$ into two spanning subgraphs $H_{1}$ and $H_{2}$ of $H^{\prime}$. For each $v \in T$, we let $A(v)$ be a set of $\frac{d_{G_{2}}(v)}{2}$ edges incident to $v$ in $H^{\prime}$. Now let

$$
V\left(H_{2}\right)=V\left(H^{\prime}\right), \quad E\left(H_{2}\right)=\bigcup_{v \in T} A(v), \quad H_{1}=H^{\prime}-E\left(H_{2}\right)
$$

From the construction and (7), for each $v \in T$,

$$
d_{H_{2}}(v)=\frac{d_{G_{2}}(v)}{2} \equiv 0 \quad(\bmod 2), \quad d_{H_{1}}(v) \geq d_{H}(v)+c-4-\frac{d_{G_{2}}(v)}{2},
$$

where $c \in\{0,1,2,3\}$ satisfies $d_{G_{1}}(v) \equiv c(\bmod 4)$. By $(6)$, we have

$$
\begin{aligned}
d_{H_{1}}(v) & \geq d_{H}(v)+c-4-\frac{d_{G_{2}}(v)}{2} \\
& \geq\left\lceil\frac{d_{G}(v)}{2}\right\rceil-1+c-4-\frac{d_{G_{2}}(v)}{2} \\
& \geq\left\lceil\frac{d_{G}(v)}{2}\right\rceil-1+c-4-\frac{1}{2}\left(\left\lfloor\frac{d_{G}(v)}{2}\right\rfloor+4-c\right) \\
& \geq \frac{d_{G}(v)}{4}-7,
\end{aligned}
$$


which is at least 2 , since $\delta(G) \geq 33$.

Let

$$
m_{1}=e\left(H_{1}\right), \quad m_{2}=e\left(G_{2}\right), \quad m_{3}=e\left(H_{2}\right) .
$$

Note that $m_{1}+m_{2}+m_{3}+\left|M^{*}\right|=m:=e(G)$. We will now give an orientation $D$ of $G$ and a labeling $\sigma$ of $A(D)$ through five parts below.

(1) Orient and label $H_{1}$ : direct each edge from $T$ to $S$. For each $i \in\left[1, n_{1}\right]$, let $A_{i}$ be the set of all edges incident to $y_{i}$ in $H_{1}$. Clearly, $\left|A_{1}\right|+\left|A_{2}\right|+\ldots+\left|A_{n_{1}}\right|=m_{1}$. By (9), $\left|A_{i}\right| \geq 2$. By Lemma 6 applied to $m_{1}$ with $t=n_{1}$ and $r_{i}=\left|A_{i}\right|$ for each $i \in[1, t]$, the set $\left\{1,2, \ldots, m_{1}\right\}$ can be partitioned into $R_{1}, R_{2}, \ldots, R_{n_{1}}$ such that for each $i \in\left[1, n_{1}\right]$, $\left|R_{i}\right|=\left|A_{i}\right|$ and $\sum_{r \in R_{i}} r \equiv 0\left(\bmod m_{1}+1\right)$ if $m_{1}$ is even, and $\sum_{r \in R_{i}} r \equiv 0\left(\bmod m_{1}\right)$ if $m_{1}$ is odd. Label edges in $A_{i}$ by integers in $R_{i}$ in an arbitrary way as long as distinct edges receive distinct labels.

(2) Orient and label $G_{2}$ : Note that for each $y \in T, d_{G_{2}}(y)$ is even and $N_{G_{2}}(y) \cap S \neq \emptyset$ by the construction of $G_{2}$. Thus, we apply Lemma 7 to get the orientation and labeling of $G_{2}$ with $p=m_{1}$ and $m=m_{2}$ with the furthermore requirement for vertices in $T$. Let $D_{2}$ be the orientation of $G_{2}$ and $\sigma_{2}$ be the labeling. We have

$$
\begin{gathered}
-\left(m_{1}+m_{2}\right)+\left\lfloor\frac{d_{G_{2}}(x)-1}{2}\right\rfloor \leq \quad s_{\left[D_{2}, \sigma_{2}\right]}(x) \leq\left\lfloor\frac{d_{G_{2}}(x)-1}{2}\right\rfloor+\left(m_{1}+m_{2}\right) \quad \text { for } x \in S, \\
s_{\left[D_{2}, \sigma_{2}\right]}(y)=\frac{d_{G_{2}}(y)}{2} \quad \text { for } y \in T .
\end{gathered}
$$

(3) Orient and label $H_{2}$ : applying Lemma 8 to get the orientation and labeling of $H_{2}$ with $p=m_{1}+m_{2}$ and $m=m_{3}$. Let $D_{3}$ be the orientation of $H_{2}$ and $\sigma_{3}$ be the labeling. We have

$$
\begin{aligned}
\left\lfloor\frac{d_{H_{2}}(x)-1}{2}\right\rfloor-\delta_{m} \leq \quad & s_{\left[D_{3}, \sigma_{3}\right]}(x) \leq\left\lfloor\frac{d_{H_{2}}(x)-1}{2}\right\rfloor+\delta_{m} \quad \text { for each } x \in S, \\
& s_{\left[D_{3}, \sigma_{3}\right]}(y)=-d_{H_{2}}(y) \quad \text { for each } y \in T,
\end{aligned}
$$

where $\delta_{m}=m_{1}+m_{2}+m_{3}$ if $m_{3} \equiv 0(\bmod 4)$, and $\delta_{m}=m_{1}+m_{2}+m_{3}+1$ if $m_{3} \equiv 2$ $(\bmod 4)$.

(4) Orient and label $M^{*} \backslash M=\left\{e_{n_{2}+1}, \ldots, e_{n_{1}}\right\}$ : direct each edge from $T$ to $S$. If $m_{3} \equiv 0$ $(\bmod 4)$, for each $i \in\left[n_{2}+1, n_{1}\right]$, assign $m_{1}+m_{2}+m_{3}+\left(i-n_{2}\right)$ to $e_{i}$. If $m_{3} \equiv 2(\bmod 4)$, assign $m_{1}+m_{2}+m_{3}$ to $e_{n_{2}+1}$, and for each $i \in\left[n_{2}+2, n_{1}\right]$, assign $m_{1}+m_{2}+m_{3}+\left(i-n_{2}\right)$ to $e_{i}$.

Let $D^{*}$ be the union of the digraphs obtained through the four parts above, and $\sigma^{*}$ be the labeling on $A\left(D^{*}\right)$ consists of the four labelings above. Assume that the sums at vertices from $S=\left\{x_{1}, \ldots, x_{n_{2}}\right\}$ satisfy

$$
s_{\left[D^{*}, \sigma^{*}\right]}\left(x_{1}\right) \leq s_{\left[D^{*}, \sigma^{*}\right]}\left(x_{2}\right) \leq \ldots \leq s_{\left[D^{*}, \sigma^{*}\right]}\left(x_{n_{2}}\right) .
$$


(5) Orient and label $M$ : direct each edge from $T$ to $S$. If $n_{1} \geq n_{2}+1$ or $m_{3} \equiv 0(\bmod 4)$, for each $i \in\left[1, n_{2}\right]$, assign $m_{1}+m_{2}+m_{3}+n_{1}-n_{2}+i$ to $x_{i} y_{i}$. If $n_{1}=n_{2}$ and $m_{3} \equiv 2$ $(\bmod 4)$, assign $m_{1}+m_{2}+m_{3}$ to $x_{1} y_{1}$, and for each $i \in\left[2, n_{2}\right]$, assign $m_{1}+m_{2}+m_{3}+i$ to $x_{i} y_{i}$.

Let $D$ and $\sigma$ be the resulting orientation and labeling, respectively. It is clear that $\sigma$ is injective. We show that $\sigma$ is an antimagic labeling of $D$.

By Step 5, we have

$$
s_{[D, \sigma]}\left(x_{1}\right)<s_{[D, \sigma]}\left(x_{2}\right)<\ldots<s_{[D, \sigma]}\left(x_{n_{2}}\right) .
$$

Furthermore, for each $i \in\left[1, n_{2}\right]$, by (10) and (11), $s_{\left[D^{*}, \sigma^{*}\right]}\left(x_{i}\right) \geq\left\lceil\frac{d_{G_{2}}\left(x_{i}\right)-1}{2}\right\rceil-m_{1}-m_{2}+$ $\left\lceil\frac{d_{H_{2}}\left(x_{i}\right)-1}{2}\right\rceil-m_{1}-m_{2}-m_{3}-1$, we know $s_{[D, \sigma]}\left(x_{i}\right) \geq s_{\left[D^{*}, \sigma^{*}\right]}\left(x_{i}\right)+m_{1}+m_{2}+m_{3} \geq-m_{1}-$ $m_{2}-1$. For each vertex $y_{i} \in T, i \in\left[1, n_{1}\right]$, for all the edges incident to $y_{i}$ that are contained in $G_{2} \cup H_{2}$, the partial sum at $y_{i}$ of the labels assigned to these edges is zero by (8), (10) and (11). All other edges incident to $y_{i}$ that are contained in $H_{1} \cup M^{*}$ are oriented towards $S$. Thus, $s_{[D, \sigma]}\left(y_{i}\right)<0$. Furthermore, by Steps 1,4 and $5, s_{[D, \sigma]}\left(y_{i}\right) \leq-m_{1}-m_{2}-m_{3}-3$.

Thus, for each $x \in S$ and each $y \in T, s_{[D, \sigma]}(x)>s_{[D, \sigma]}(y)$. Therefore, it is left to only show that all vertices from $T$ have distinct sums under $\sigma$ in $D$.

By Steps $1,4,5$, and (10) and (11), for each $i \in\left[1, n_{1}\right]$ and some integer $a_{i} \geq 1$, we have

$$
\left|s_{[D, \sigma]}\left(y_{i}\right)\right|= \begin{cases}\frac{d_{G_{2}}\left(y_{i}\right)}{2}-d_{H_{2}}\left(y_{i}\right)+a_{i} m_{1}+m_{1}+m_{2}+m_{3}+\sigma_{i}, & \text { if } m_{1} \text { is odd, } \\ \frac{d_{G_{2}}\left(y_{i}\right)}{2}-d_{H_{2}}\left(y_{i}\right)+a_{i}\left(m_{1}+1\right)+m_{1}+m_{2}+m_{3}+\sigma_{i}, & \text { if } m_{1} \text { is even }\end{cases}
$$

where $\sigma_{i} \in\left[1, n_{1}\right]$ are all distinct, and $\frac{d_{G_{2}}\left(y_{i}\right)}{2}-d_{H_{2}}\left(y_{i}\right)=0$. Since $m_{1} \geq 2 n_{1}>n_{1}$ by (9), for any two distinct $i, j \in\left[1, n_{1}\right]$,

$$
s_{[D, \sigma]}\left(y_{i}\right)-s_{[D, \sigma]}\left(y_{j}\right) \not \equiv\left\{\begin{array}{lll}
0 & \left(\bmod m_{1}\right), & \text { if } m_{1} \text { is odd } \\
0 & \left(\bmod m_{1}+1\right), & \text { if } m_{1} \text { is even }
\end{array}\right.
$$

Consequently, $s_{[D, \sigma]}\left(y_{i}\right) \neq s_{[D, \sigma]}\left(y_{j}\right)$.

The proof is now complete.

\section{References}

[1] N. Alon, G. Kaplan, A. Lev, Y. Roditty, and R. Yuster. Dense graphs are antimagic. J. Graph Theory, 47(4):297-309, 2004.

[2] Kristóf Bérczi, Attila Bernáth, and Máté Vizer. Regular graphs are antimagic. Electron. J. Combin., 22(3):Paper 3.34, 6, 2015.

[3] Feihuang Chang, Yu-Chang Liang, Zhishi Pan, and Xuding Zhu. Antimagic labeling of regular graphs. J. Graph Theory, 82(4):339-349, 2016.

[4] Daniel W. Cranston. Regular bipartite graphs are antimagic. J. Graph Theory, 60(3):173-182, 2009. 
[5] Daniel W. Cranston, Yu-Chang Liang, and Xuding Zhu. Regular graphs of odd degree are antimagic. J. Graph Theory, 80(1):28-33, 2015.

[6] Tom Eccles. Graphs of large linear size are antimagic. J. Graph Theory, 81(3):236-261, 2016.

[7] Nora Hartsfield and Gerhard Ringel. Pearls in graph theory. Academic Press, Inc., Boston, MA, 1994. A comprehensive introduction, Revised reprint of the 1990 original.

[8] Dan Hefetz. Anti-magic graphs via the combinatorial nullstellensatz. J. Graph Theory, 50(4):263-272, 2005.

[9] Dan Hefetz, Torsten Mütze, and Justus Schwartz. On antimagic directed graphs. J. Graph Theory, 64(3):219-232, 2010.

[10] Dan Hefetz, Annina Saluz, and T. T. Tran Huong. An application of the combinatorial Nullstellensatz to a graph labelling problem. J. Graph Theory, 65(1):70-82, 2010.

[11] Gil Kaplan, Arieh Lev, and Yehuda Roditty. On zero-sum partitions and anti-magic trees. Discrete Math., 309(8):2010-2014, 2009.

[12] Tong Li, Zi-Xia Song, Guanghui Wang, Donglei Yang, and Cun-Quan Zhang. Antimagic orientations of even regular graphs, 2017.

[13] Songling Shan and Xiaowei Yu. Antimagic orientation of biregular bipartite graphs. Electron. J. Combin., 24(4):Paper 4.31, 20, 2017.

[14] Chen Song and Rong-Xia Hao. Antimagic orientations of disconnected even regular graphs. Discrete Math., 342(8):2350-2355, 2019.

[15] Zi-Xia Song, Donglei Yang, and Fangfang Zhang. Antimagic orientations of graphs with given independence number. arXiv:1909.10928v2, 2019.

[16] Donglei Yang. A note on antimagic orientations of even regular graphs. Discrete Appl. Math., 267:224-228, 2019.

[17] Donglei Yang, Joshua Carlson, Andrew Owens, K. E. Perry, Inne Singgih, Zi-Xia Song, Fangfang Zhang, and Xiaohong Zhang. Antimagic orientations of graphs with large maximum degree. arXiv:908.06072v2, 2019.

[18] Zelealem B. Yilma. Antimagic properties of graphs with large maximum degree. $J$. Graph Theory, 72(4):367-373, 2013.

[19] Xiaowei Yu, Yulin Chang, and Shan Zhou. Antimagic orientation of Halin graphs. Discrete Math., 342(11):3160-3165, 2019. 